\title{
Twelve new polymorphic microsatellite markers from the loggerhead sea turtle (Caretta caretta) and cross-species amplification on other marine turtle species
}

\author{
Catalina Monzón-Argü ello $\mathbb{E}$ Joaquín Muñ oz $\mathbb{1}$ \\ Adolfo Marco $\llbracket$ Luis Felipe López-Jurado \& \\ Ciro Rico
}

\begin{abstract}
We describe 12 new polymorphic dinucleotide microsatellite loci and multiplex Polymerase Chain Reaction conditions from the loggerhead sea turtle Caretta caretta. Levels of polymorphism were assessed in 50 individuals from the nesting population of the Cape Verde Islands. Number of alleles ranged from 3 to 13 (average of 7.33) and the values of observed heterozygosities from 0.32 to 0.80 (average of 0.61 ). Cross-species amplification on three other marine turtles, Chelonia mydas, Eretmochelys imbricata and Dermochelys coriacea, revealed polymorphism and variability at eight, eleven and three loci, respectively.
\end{abstract}

Keywords Microsatellites Caretta caretta Marine turtle Dinucleotide repeats

The globally endangered loggerhead sea turtle Caretta caretta inhabits in warm temperate and tropical waters in all the ocean basins. Although the use of genetics as a tool for studying and understanding the marine turtles' biology

C. Monzón-Argüello ( $\&$ )

Instituto Canario de Ciencias Marinas, Crta. De Taliarte $s / n$, 35200 Telde, Gran Canaria, Spain

e-mail: catalinama@iccm.rcanaria.es

J. Muñoz A. Marco C. Rico

Estación Biológica de Doñana (CSIC), Avda. María Luisa s/n, 41013 Sevilla, Spain

L. F. López-Jurado

Dpto de Biología Animal, Universidad de Las Palmas de G.C., Campus de Tafira, 35017 Las Palmas de Gran Canaria, Gran

Canaria, Spain is well established, most surveys have analysed the population structure revealed by mitochondrial DNA (mtDNA) (Encalada et al. 1998; Laurent et al. 1998; Kaska 2000; Bowen et al. 2004; Carreras et al. 2006). Nuclear markers such as microsatellites have allowed the identification of conservation and management units, the differences in the migratory behaviour of males and females, and the mating behaviour of several marine turtle species (Crim et al. 2002; Bowen et al. 2005; Jensen et al. 2006). However, these studies have been limited by the use of a few microsatellites, non-specific in many cases, which are conserved across all cheloniid turtles (Fitzsimmons et al. 1995). Recently, 15 specific tetranucleotide microsatellites for Caretta caretta have been published (Shamblin et al. 2007). Here, we present 12 new polymorphic specific dinucleotide microsatellite markers that could be useful in future population genetic studies from nesting and foraging areas of the loggerhead sea turtle. In addition, cross-species amplification of these $\mathrm{C}$. caretta microsatellite primers was performed on three other marine turtle species using between 6 and 8 individuals per species.

Microsatellite markers were identified by the development of an enriched genomic library as described by Glenn and Schable (2003). Whole genomic DNA was extracted from tissue samples stored in ethanol 96\% of four C. caretta individuals using Qiamp Kit (QIAGEN). We digested the isolated DNA with RsaI restriction enzyme (SIGMA) and the DNA fragments were ligated to SNX linkers using T4-DNA ligase (BioLabs). Ligated DNA was enriched with a biotinylated probe mixture consisting of $(\mathrm{GT})_{10}$ and $(\mathrm{CT})_{10}$. DNA fragments with repetitive sequences were captured by streptavidin-coated Dynabeads (Oxoid) and separated by a magnetic field. JM109 High Efficiency Competent Cells (Invitrogen) were transformed with the recovered enriched DNA using 
Table 1 Details of the 12 microsatellite markers developed in the study

\begin{tabular}{|c|c|c|c|c|c|c|c|c|c|c|c|c|}
\hline Locus & GB Accession no. & Repeat motif & Primer sequence $\left(5^{0}-3^{0}\right)$ & $\mathrm{N}$ & Size Range (bp) & NA & $\mathrm{H}_{\mathrm{O}}$ & $\mathrm{H}_{\mathrm{E}}$ & PIC & MP & $\mathrm{Mg}(\mathrm{mM})$ & $\mathrm{P}(\mathbf{1 M})$ \\
\hline Cc-2 & EF679214 & $(\mathrm{TA})_{4}(\mathrm{GA})_{13}$ & $\begin{array}{l}\text { F PETCCCССАTAACACCACATCTC } \\
\text { R AGGTCACAAATGGAGCAAGC }\end{array}$ & 50 & $225-233$ & 3 & 0.420 & 0.444 & 0.382 & 2 & 3.5 & 0.10 \\
\hline Сc-8 & EF679216 & $(\mathrm{GT})_{8}(\mathrm{AT})(\mathrm{GT})_{4}$ & $\begin{array}{l}\text { F PETTGATGGAAACCCCTTCAAAC } \\
\text { R TGTCACGGAGACACAAACATT }\end{array}$ & 50 & 182-204 & 6 & 0.560 & 0.561 & 0.527 & 2 & 3.5 & 0.10 \\
\hline Сc-10 & EF679217 & $(\mathrm{CT})_{13}$ & $\begin{array}{l}\text { F FAMTCCACATGGGGTTGTATGAA } \\
\text { R TGCCCTCCTTGAGAATTCAG }\end{array}$ & 50 & $415-431$ & 4 & 0.700 & 0.684 & 0.630 & 2 & 3.5 & 0.10 \\
\hline Cc-13 & EF679218 & $(\mathrm{CA})_{17}$ & $\begin{array}{l}\text { F NEDCACCATCTGGGGAGATGAAT } \\
\text { R AGGCTTGCCTTTTTGAAGGT }\end{array}$ & 50 & $402-421$ & 13 & 0.720 & 0.777 & 0.751 & 1 & 3 & 0.15 \\
\hline Cc-16 & EF679219 & $(\mathrm{CT})_{16}$ & $\begin{array}{l}\text { F NEDGTCTTGACTGACCTGGCACA } \\
\text { R CCACCAAAAGGAGCAAAATG }\end{array}$ & 50 & $342-354$ & 6 & 0.760 & 0.750 & 0.702 & 1 & 3 & 0.15 \\
\hline Сc-17 & EF679220 & $(\mathrm{AG})_{16}$ & $\begin{array}{l}\text { F FAMCCACTGGAAGTCTAAGAAGAGTGC } \\
\text { R GGAATTGAAGGGATTTTGCT }\end{array}$ & 50 & $320-348$ & 11 & 0.760 & 0.740 & 0.696 & 3 & 3 & 0.20 \\
\hline Сc-21 & EF679221 & $(\mathrm{TG})_{17}(\mathrm{AG})_{8}$ & $\begin{array}{l}\text { F VICGGCTGTTCCTCTGAAACCTG } \\
\text { R AGCAATGACAAGGGAGCATT }\end{array}$ & 50 & $374-410$ & 11 & 0.620 & 0.675 & 0.650 & 2 & 3.5 & 0.10 \\
\hline Cc-22 & EF679222 & $(\mathrm{GA})_{17}$ & $\begin{array}{l}\text { F VICCCСCСАCTGCTTTAACTTCA } \\
\text { R TATTCCAACATGCCCACAGA }\end{array}$ & 50 & 218-236 & 6 & 0.500 & 0.543 & 0.493 & 1 & 3 & 0.15 \\
\hline Сc-25 & EF679223 & $(\mathrm{CT})_{15}$ & $\begin{array}{l}\text { F PETTTTTGCTTTCCCCATCTGAC } \\
\text { R AGCCTCCAGCACAGCATTAT }\end{array}$ & 50 & $320-344$ & 9 & 0.800 & 0.775 & 0.733 & 2 & 3.5 & 0.10 \\
\hline Сc-28 & EF679224 & $(\mathrm{CA})_{16}$ & $\begin{array}{l}\text { F FAMAGCCCATATGTTTCCCTTCA } \\
\text { R TTGGCCCATCTTATTTCAGTG }\end{array}$ & 50 & 191-211 & 7 & 0.520 & 0.539 & 0.486 & 3 & 3 & 0.20 \\
\hline Сc-30 & EF679225 & $(\mathrm{CT})_{13}(\mathrm{CA})(\mathrm{CT})_{21}$ & $\begin{array}{l}\text { F NEDCTTTTGGAGGCAGGCTAGTG } \\
\text { R GAAGCCAGGTTGATCAGGAG }\end{array}$ & 50 & 161-189 & 9 & 0.680 & 0.798 & 0.762 & 4 & 3 & 0.20 \\
\hline Сc-32 & EF679226 & $(\mathrm{TC})_{11}$ & $\begin{array}{l}\text { F PETGCACATCTGGCTGTGAGAAG } \\
\text { R CCСССTCACCTCAGTAGCTT }\end{array}$ & 50 & $386-390$ & 3 & 0.320 & 0.381 & 0.325 & 4 & 3 & 0.10 \\
\hline $\begin{array}{l}\text { GB Acc } \\
\mathrm{H}_{\mathrm{E}}-\mathrm{ex} \\
\text { primers }\end{array}$ & $\begin{array}{l}\text { no.-GenBank Acc } \\
\text { pected heterozygosit } \\
\text { concentration }\end{array}$ & ; PIC-polymorphic i & $\begin{array}{l}\text { nber of samples analyzed; Size range-predicted s } \\
\text { formation content; MP—-numbers indicate those } 1\end{array}$ & ci w & $\begin{array}{l}\text { he PCR product an } \\
\text { ch share multiplex }\end{array}$ & $\begin{array}{l}\text { plifiec } \\
\text { PCR }\end{array}$ & NA-n & umber of & alleles; & $\begin{array}{l}\mathrm{H}_{0}-\mathrm{C} \\
\mathrm{l}_{2} \mathrm{col}\end{array}$ & $\begin{array}{l}\text { served heter } \\
\text { entration an }\end{array}$ & zygosity; \\
\hline
\end{tabular}


Table 2 Summary of the cross-species amplifications of loggerhead sea turtle specific microsatellite markers tested on three marine turtle species: green turtle (Chelonia mydas), hawksbill turtle (Eretmochelys imbricata) and leatherback (Dermochelys coriacea)

\begin{tabular}{|c|c|c|c|c|c|c|c|c|}
\hline Locus & Spp. & $\mathrm{N}$ & Status & NA & Size range (bp) & $\mathrm{T}^{\mathrm{a}}\left({ }^{\circ} \mathrm{C}\right)$ & $\mathrm{Mg}(\mathrm{mM})$ & $\mathrm{P}(\mathbf{1} \mathrm{M})$ \\
\hline \multirow[t]{3}{*}{ Сc-1 } & C. mydas & 6 & $\mathrm{p}$ & 2 & $168-170$ & 61 & 3 & 0.15 \\
\hline & E. imbricata & 8 & $\mathrm{p}$ & 6 & 159-181 & 61 & 3 & \\
\hline & D. coriacea & 7 & $\mathrm{~m}$ & 1 & 175 & 55 & 3.5 & \\
\hline \multirow[t]{3}{*}{ Cc-2 } & C. mydas & 6 & $\mathrm{p}$ & 4 & 213-231 & 61 & 3.5 & 0.15 \\
\hline & E. imbricata & 8 & $\mathrm{p}$ & 6 & 219-231 & 61 & 3.5 & \\
\hline & D. coriacea & 7 & $\mathrm{~m}$ & 1 & 221 & 61 & 3.5 & \\
\hline \multirow[t]{3}{*}{ Сc-6 } & C. mydas & 6 & $\mathrm{p}$ & 6 & 188-206 & 61 & 3 & 0.15 \\
\hline & E. imbricata & 8 & $\mathrm{p}$ & 2 & 216-222 & 61 & 3 & \\
\hline & D. coriacea & 7 & $\mathrm{~m}$ & 1 & 160 & 52 & 3.5 & \\
\hline \multirow[t]{3}{*}{ Cc-10 } & C. mydas & 6 & $\mathrm{p}$ & 5 & $407-421$ & 61 & 3.5 & 0.15 \\
\hline & E. imbricata & 8 & $\mathrm{p}$ & 2 & 376-402 & 61 & 3.5 & \\
\hline & D. coriacea & 7 & na & 0 & - & $(52-61)$ & 3.5 & \\
\hline \multirow[t]{3}{*}{ Cc-13 } & C. mydas & 6 & $\mathrm{p}$ & 6 & 399-431 & 61 & 3 & 0.15 \\
\hline & E. imbricata & 8 & $\mathrm{p}$ & 6 & $391-416$ & 61 & 3 & \\
\hline & D. coriacea & 7 & $\mathrm{~m}$ & 1 & 388 & 61 & 3 & \\
\hline \multirow[t]{3}{*}{ Cc-16 } & C. mydas & 6 & na & 0 & - & $(52-61)$ & 3 & 0.15 \\
\hline & E. imbricata & 8 & $\mathrm{p}$ & 2 & $326-340$ & 61 & 3 & \\
\hline & D. coriacea & 7 & na & 0 & - & $(52-61)$ & 3 & \\
\hline \multirow[t]{3}{*}{ Сc-17 } & C. mydas & 6 & $\mathrm{~m}$ & 1 & 321 & 61 & 3 & 0.15 \\
\hline & E. imbricata & 8 & $\mathrm{p}$ & 7 & $323-355$ & 61 & 3 & \\
\hline & D. coriacea & 7 & $\mathrm{p}$ & 2 & $316-318$ & 61 & 3 & \\
\hline \multirow[t]{3}{*}{ Сc-21 } & C. mydas & 6 & na & 0 & - & $(52-61)$ & 3 & 0.15 \\
\hline & E. imbricata & 8 & na & 0 & - & $(52-61)$ & 3 & \\
\hline & D. coriacea & 7 & $\mathrm{~m}$ & 1 & 388 & 61 & 3.5 & \\
\hline \multirow[t]{3}{*}{ Сc-22 } & C. mydas & 6 & $\mathrm{p}$ & 7 & 210-230 & 61 & 3 & 0.15 \\
\hline & E. imbricata & 8 & $\mathrm{p}$ & 3 & 228-232 & 61 & 3 & \\
\hline & D. coriacea & 7 & $\mathrm{p}$ & 2 & 219-223 & 61 & 3 & \\
\hline \multirow[t]{3}{*}{ Cc-25 } & C. mydas & 6 & na & 0 & - & $(52-61)$ & 3.5 & 0.15 \\
\hline & E. imbricata & 8 & $\mathrm{p}$ & 2 & $320-322$ & 61 & 3.5 & \\
\hline & D. coriacea & 7 & $\mathrm{~m}$ & 1 & 322 & 61 & 3.5 & \\
\hline \multirow[t]{3}{*}{ Cc-28 } & C. mydas & 6 & $\mathrm{p}$ & 3 & 188-198 & 61 & 3 & 0.15 \\
\hline & E. imbricata & 8 & $\mathrm{p}$ & 6 & $198-224$ & 61 & 3 & \\
\hline & D. coriacea & 7 & $\mathrm{p}$ & 2 & 194-196 & 61 & 3 & \\
\hline \multirow[t]{3}{*}{ Cc-30 } & C. mydas & 6 & $\mathrm{p}$ & 8 & 165-201 & 61 & 3 & 0.15 \\
\hline & E. imbricata & 8 & $\mathrm{p}$ & 4 & 157-193 & 61 & 3 & \\
\hline & D. coriacea & 7 & $\mathrm{~m}$ & 1 & 154 & 61 & 3 & \\
\hline \multirow[t]{3}{*}{ Cc-32 } & C. mydas & 6 & $\mathrm{~m}$ & 1 & 382 & 61 & 3 & 0.15 \\
\hline & E. imbricata & 8 & $\mathrm{~m}$ & 1 & 382 & 61 & 3 & \\
\hline & D. coriacea & 7 & $\mathrm{~m}$ & 1 & 357 & 61 & 3 & \\
\hline
\end{tabular}

Spp.—species name; N—number of samples analyzed; NA—number of alleles; Size range—predicted size of the PCR product amplified; $\mathrm{T}^{\mathrm{a}}$ — annealing temperature, in brackets range of temperature used; $\mathrm{Mg}$ - final $\mathrm{MgCl}_{2}$ concentration; $\mathrm{P}$-final primers concentration. In the column Status we show: na—no amplification; p-polymorphic; and m-monomorphic

the cloning vector pGEM-T Easy Vector II (Promega). We amplify a total of 223 positive colonies (white) using the universal M13 primers. PCR products ranging between 500 and 1000 base pair (bp) were sequenced using Big Dye Terminator ver. 3.1 chemistry (Applied Biosystems) in an ABI 3100 automated sequencer. Chromatograms were analyzed and edited using BioEdit Sequence Alignment Editor ver. 7.0.9 (Hall 1999) 
screening for microsatellite repeats. We designed 23 primer pairs using Primer3 v.0.3.0 online software (Rozen and Skaletsky 2000) out of which 12 consistently amplified and were polymorphic.

Four multiplex PCR amplifications were performed in a 2011 total volume with $40 \mathrm{ng}$ of genomic DNA. Final concentrations for primers and $\mathrm{MgCl}_{2}$ for each reaction are shown in Table 1. Final concentrations for optimizing reactions were PCR buffer 19 (Bioline), 20 1M BSA, $0.25 \mathrm{IM}$ dNTPs, and 0.5 U Taq DNA polymerase (Bioline). Reactions for all loci were carried out using the same thermal cycling program: an initial denaturation at $94^{\circ} \mathrm{C}$ for $5 \mathrm{~min}, 30$ cycles of $94^{\circ} \mathrm{C}$ for $30 \mathrm{~s} 61^{\circ} \mathrm{C}$ for $30 \mathrm{~s}$ and $72^{\circ} \mathrm{C}$ for $30 \mathrm{~s}$, and a final extension period of $10 \mathrm{~min}$ at $72^{\circ} \mathrm{C}$. Following this procedure, we genotyped 50 individuals of C. caretta from the Cape Verde population. Fragment sizes were scored using an ABI 3100 automated sequencer with LIZ 500 (Applied Biosystems) as internal fluorescent size standard. The results were analysed using GENEMAPPER ver. 3.5. We calculated observed and expected heterozygosities, number of alleles per locus and polymorphic information content (PIC) with Cervus ver. 3.0 (Marshall et al. 1998). We also tested for linkage disequilibrium and deviations from Hardy-Weinberg equilibrium using Arlequin 3.0 program (Excoffier et al. 2005). Sequential Bonferroni corrections were further conducted using a global $\mathrm{P}$ value of 0.05 (Rice 1989).

In addition to the 12 polymorphic loci obtained for the loggerhead sea turtle (Table 1), two more loci Cc-1 (GenBank Accession Number EF679213) and Cc-6 (GB Acc. Num. EF679215) were tested in 16 loggerhead sea turtle samples from the Canary Islands feeding ground, a mixed stock of different populations. We found only one allele for Cc- 1 and two alleles, one of them in a frequency of $94 \%$, for Cc- 6 . However, these two loci resulted to be polymorphic for other marine turtle species (Table 2). Table 1 shows locus designation, GenBank Accession Numbers, repeat motifs, primer sequences, allele attributes, PIC estimates and information about PCR conditions. In spite of being a dinucleotide marker, Cc-13 presented alleles that differ only by one base. This could be caused by the fact that this locus contains two additional STR sequences of a multiple adenine in one and a multiple cytosine in the other. After sequential Bonferroni corrections, no loci showed significant deviations of HardyWeinberg equilibrium, but two pair of loci yielded significant linkage disequilibrium (Cc-21 with Cc-28 and Cc-21 with Cc-32). Table 2 summarizes the polymorphic microsatellite results of the cross-species amplification tests. In conclusion, we identified 12 new specific polymorphic microsatellite markers for the loggerhead sea turtle which should prove useful in population and parentage analysis in this and other marine turtle species.
Acknowledgements We thank to Pascual Calabuig, Nuria Varo, Ana Liria, Pedro López, and Paula Sanz for their contributions on sampling and field collection. Miguel Alcaide and Carlos Carreras helped us during laboratory procedures and the genomic library development. We are very grateful to Instituto Canario de Ciencias Marinas, Estación Biológica de Doñana, Junta de Andalucía, and the Cape Verde government for helping with the field and laboratory equipment. CMA was supported by a $\mathrm{PhD}$ grant from the Canary Islands government.

\section{References}

Bowen BW, Bass AL, Chow S-M, Bostrom M, Bjorndal KA, Bolten AB, Okuyama T, Bolker BM, Epperly S, Lacasella E, Shaver D, Dodd M, Hopkins-Murphy SR, Musick JA, Swingle M, RankinBaransky K, Teas W, Witzell WN, Dutton PH (2004) Natal homing in juvenile loggerhead turtles (Caretta caretta). Mol Ecol 13:3797-3808

Bowen BW, Bass AL, Soares L, Toonen J (2005) Conservation implications of complex population structure: lessons from the loggerhead turtle (Caretta caretta). Mol Ecol 14:2389-2402

Carreras C, Pon S, Maffucci F, Pascual M, Barceló A, Bentivegna F, Cardona L, Alegre F, SanFélix M, Fernández G, Aguilar A (2006) Genetic structuring of immature loggerhead sea turtles (Caretta caretta) in the Mediterranean Sea reflects water circulation patterns. Mar Biol 149(5):1269-1279

Crim JL, Spotila LD, Spotila JR, O' Connor M, Reina R, Williams CJ, Paladinos FV (2002) The leatherback turtle, Dermochelys coriacea, exhibits both polyandry and polygyny. Mol Ecol 11:2097-2106

Encalada SE, Bjorndal KA, Bolten AB, Zurita JC, Schroeder B, Possardt E, Sears CJ, Bowen BW (1998) Population structure of loggerhead turtle (Caretta caretta) nesting colonies in the Atlantic and Mediterranean as inferred from mitochondrial DNA control region sequences. Marine Biology 130:567-575

Excoffier L, Laval G, Schneider S (2005) Arlequin (version 3.0): An integrated software package for population genetics data analysis. Evol Bioinform Online 1:47-50

FitzSimmons NN, Moritz C, Moore SS (1995) Conservation and dynamics of microsatellite loci over 300 million years of marine turtle evolution. Mol Biol Evol 12(3):432-440

Glenn TC, Schable M (2003) Microsatellite Isolation with Dynabeads 2003@. http://www.uga.edu/srel/DNA_Lab/protocols.htm

Hall TA (1999) BioEdit: a user-friendly biological sequence alignment editor and analysis program for Windows 95/98/NT. Nucl. Acids Symp Ser 41:95-98

Jensen MP, Abreu-Grobois FA, Frydenberg J, Loeschcke V (2006) Microsatellites provide insight into contrasting mating patterns in arribada vs. non-arribada olive ridley sea turtle rookeries. Mol Ecol 15(9):2567-2575

Kaska Y (2000) Genetic structure of Mediterranean sea turtle populations. Turk J Zool 24:191-197

Laurent L, Casale P, Bradai MN, Godley BJ, Gerosa G, Broderick AC, Schroth W, Scierwater B, Levy AM, Freggi D, Abd El-Mawla EM, Hadoud DA, Gomati HE, Domingo M, Hadjichristophorou M, Kornaraky L, Demirayak F, Gautier CH (1998) Molecular resolution of marine turtle stock composition in fishery by-catch: a case study in the Mediterranean. Mol Ecol 7:1529-1542

Marshall TC, Slate J, Kruuk LEB, Pemberton JM (1998) Statistical confidence for likelihood-based paternity inference in natural populations. Mol Ecol 7:639-655

Rice WR (1989) Analyzing tables of statistical tests. Evolution 43:223-225 
Rozen S, Skaletsky HJ (2000) Primer 3 on the www for general users and for biologist programmers. In: Krawetz S, Misener S (eds) Bioinformatics methods and protocols: Methods in molecular biology. Humana Press, Totowa, NJ, pp 365-386
Shamblin BM, Faitcloth BC, Dodd M, Wood-Jones A, Castleberry SB, Carroll JP, Nairn CJ (2007) Tetranucleotide microsatellites from the loggerhead sea turtle (Caretta caretta). Mol Ecol Notes 7:784-787 